\section{JTI}

JOURNAL OF

TRAUMA AND INJURY

\title{
The Suitability of the CDC Field Triage for Korean Trauma Care
}

\author{
Kang Kook Choi, M.D., Myung Jin Jang, M.S., Min A Lee, M.D., Gil Jae Lee, M.D., \\ Byungchul Yoo, M.D., Youngeun Park, M.D., Jung Nam Lee, M.D. \\ Department of Traumatology, Gachon University College of Medicine, Gachon University \\ Gil Medical Center, Incheon, Korea
}

Purpose: Accurate and appropriate prehospital field triage is essential for a trauma

Received: March 20, 2020

Revised: March 23, 2020

Accepted: March 24, 2020

\section{Correspondence to}

Jung Nam Lee, M.D.

Department of Traumatology, Gachon University College of Medicine, Gachon

University Gil Medical Center, 783

Namdong-daero, Namdong-gu, Incheon

21556, Korea

Tel: $+82-32-460-3010$

Fax: +82-32-460-2372

E-mail:jnlee@gilhospital.com

This paper was presented at 2018 6th Pan Pacific Trauma Congress. system. The Korean trauma system (established in 2014) uses the trauma field triage algorithm of the United States Centers for Disease Control and Prevention (CDC). This study evaluated the suitability of the CDC field triage criteria for major trauma cases (injury severity score $>15$ ) in Korea.

Methods: This retrospective cohort study evaluated trauma patients who presented at the authors' regional trauma center from January 1 to May 7, 2017. The undertriage and overtriage rates of each CDC field triage step were calculated. Receiver operating characteristic curves were constructed, and the area under the curve (AUC) was evaluated for each step.

Results: Among the 1,009 enrolled patients, 168 (16.7\%) had major trauma. The undertriage/overtriage rates of each step (steps I, II, III, and IV) of CDC field triage were $9.2 \% / 47.4 \%, 6.3 \% / 50.8 \%, 4.5 \% / 59.4 \%$, and $5.3 \% / 78.9 \%$, respectively. The AUC values of each CDC triage step were $0.722,0.783,0.791$, and 0.615 , respectively. The AUC values of the separate components of each step (physiologic criteria, anatomic criteria, mechanism-of-injury criteria, and special considerations) were $0.722,0.648,0.647$, and 0.456 , respectively.

Conclusions: The CDC field triage system is acceptable, but not ideal, for Korean trauma care. If we follow the protocol, it would be preferable to omit step IV. The Korean Triage and Acuity Scale may be a good indicator for in-hospital triage. However, a new triage protocol that is simple to estimate on-scene while having good performance should be developed.

Keywords: Wounds and injuries; Triage; Injury severity score; Emergency medical services; Health resources

(http://creativecommons.org/licenses/by-nc/4.0/) which permits unrestricted noncommercial use, distribution, and reproduction in any medium, provided the original work is properly cited. 


\section{INTRODUCTION}

Accurate and appropriate prehospital field triage is especially important in trauma care, compared to other emergency medical areas. This is because failure of triage in trauma care can result in critical problems. Undertriage is the most unfavorable aspect of inappropriate prehospital triage in trauma care, because it can lead to delayed or missed interventions, thereby resulting in morbidity and mortality in a critically ill patient [1-3]. However, overtriage can also be a burden because the available resources for trauma care are limited. Therefore, overtriage can disrupt the management of a critically ill patient. Prehospital overtriage causes an increase in expenses, geographic constraints on patient treatment, and overcrowding at referral centers [4]. As reported in previous studies, good field triage meets an undertriage rate of $<5 \%$ with an overtriage rate of $25-50 \%$ [3,5]. Due to its importance, several countries have developed their own trauma field triage systems, starting with the United States $[4,6]$.

Korea does not have enough accumulated data to create its own trauma field triage system, because the Korean trauma system was only established in 2014. Currently, Korea uses the Standard Protocols for 119 Emergency Medical Services Providers (Korean trauma field triage; KTF) [7], which is almost the same as the trauma field triage algorithm of the United States developed by the Centers for Disease Control and Prevention (CDC) [3]. As more than 5 years have passed since the Korean trauma system was launched, we think that the time has come to examine the suitability of the CDC field triage system for domestic cases of trauma care.

Korea also utilizes the Korean Triage and Acuity Scale (KTAS), which was developed in accordance with the Canadian Triage and Acuity Scale for the triage of emergency patients, not limited to trauma [8]. The KTAS has been used since 2016 in Korea, but no previous study has investigated the suitability of this triage system for Korean trauma patients.

Therefore, the aim of this study was to evaluate suitability of the CDC field triage and KTAS criteria for cases of major trauma, which was defined by an injury severity score (ISS) of $>15$ [9]. The ISS is the most frequently used indicator at hospitals to assess the severity of trauma [6].

\section{METHODS}

This retrospective cohort study evaluated trauma patients who presented at the Gil Medical Center which is the regional trauma center of Incheon, Korea from January 1 to May 7, 2017 using the Korean Trauma Data Base (KTDB). Patients with backgrounds of drowning, choking, hanging, sexual assault, and burns were excluded. A total of 1,008 trauma patients were ultimately enrolled in this study.

There are only slight differences between the CDC field triage system and the KTF, reflecting the following changes that were made to adjust the system to use metric units: the 20-feet fall criterion was changed to $6 \mathrm{~m}, 10$ feet was changed to $3 \mathrm{~m}, 12$ inches (in the context of a highrisk auto crash) was changed to $30 \mathrm{~cm}$, and $20 \mathrm{mph}$ was changed to $30 \mathrm{~km} / \mathrm{h}[7]$.

Patient variables were collected using data from both prehospital medical providers and our medical records, in accordance with each step of the CDC trauma field triage system. The event was considered nonexistent for variables with missing descriptions in both records. Step I includes sex, age, and initial physiologic signs, such as initial systolic blood pressure, initial respiratory rate, initial Glasgow Coma Scale (GCS), initial pulse rate, and initial hypotension (systolic blood pressure $<90 \mathrm{mmHg}$ ). In step II, penetrating injury, flail chest, two or more proximal long-bone fractures, crushed/degloved/mangled extremities, amputation above the ankle or wrist, pelvic fracture, open or depressed skull fracture, and paralysis were included. Step III includes fall height, high-risk auto crash (intrusion of car $>30 \mathrm{~cm}$, ejection, death as passenger), auto versus pedestrian/bicyclist thrown or run over at $>30 \mathrm{~km} / \mathrm{h}$, and motorcycle crash $>30 \mathrm{~km} / \mathrm{h}$. Step IV includes age, anticoagulation status or bleeding disorders, burns, time-sensitive extremity injuries, end-stage renal disease requiring dialysis, pregnancy $>20$ weeks, and emergency medical service (EMS) provider judgment. The ISS for every patient was examined by a program manager from the Department of Traumatology using the 2005 Abbreviated Injury Scale (update 2008) [9]. The KTAS scores of each patient were collected using the KTDB. The KTAS score is presented by a qualified nurse as soon as the patient enters the emergency room. 
The undertriage and overtriage rates of each step of CDC field triage were calculated using the Cribari matrix [10]. The KTAS criteria were as follows: KTAS $\leq 1$, KTAS $\leq 2$, KTAS $\leq 3$, KTAS $\leq 4$, and KTAS $\leq 5$, and then the undertriage and overtriage rates of each categorization were evaluated. Receiver operating characteristic (ROC) curves were constructed, and the area under the curve (AUC) was evaluated for each step of the criteria. ROC curves were also constructed for the separate components of each step (physiologic criteria, anatomic criteria, mechanism-of-injury criteria, and special considerations). All statistical analyses were conducted using SPSS version 19.0 for Windows (IBM Corp., Armonk, NY, USA).

\section{RESULTS}

The mean age of the enrolled patients was $50.7 \pm 21.6$ years. The male-to-female ratio was 1.75 (643/366). There were 738 cases of direct transfer from the scene (74.1\%) and 261 cases of transfer from other hospitals. Among the 1,009 enrolled patients, 168 were major trauma patients (16.7\%). The undertriage/overtriage rates of each step (steps I, II, III, and IV) of the CDC field triage system were as follows: $9.2 \% / 47.4 \%, 6.3 \% / 50.8 \%, 4.5 \% / 59.4 \%$, and 5.3\%/78.9\%, respectively, as illustrated in Table 1. The undertriage/overtriage rates of each KTAS criterion $(\mathrm{KTAS} \leq 1, \mathrm{KTAS} \leq 2, \mathrm{KTAS} \leq 3, \mathrm{KTAS} \leq 4$, and $\mathrm{KTAS} \leq 5)$ were as follows: $15.2 \% / 50.0 \%, 7.4 \% / 57.5 \%, 4.1 \% / 79.9 \%$, $0 \% / 83.3 \%$, and $0 \% / 83.3 \%$, respectively (Table 2 ). The AUC values of each step (steps I, II, III, and IV) of the CDC triage system were $0.722,0.783,0.791$, and 0.615 , respectively (Fig. 1). The AUC values of the separate components (physiologic criteria, anatomic criteria, mechanism-of-injury criteria, and special considerations) of

Table 1. The undertriage/overtriage rate of each step of the CDC field triage system

\begin{tabular}{|llccc|}
\hline & Step I & Step II & Step III & Step IV \\
\hline Undertriage & $77(9.2)$ & $48(6.3)$ & $30(4.5)$ & $15(5.3)$ \\
Overtriage & $82(47.4)$ & $124(50.8)$ & $202(59.4)$ & $572(78.9)$ \\
\hline
\end{tabular}

Values are presented as number (\%).

CDC: Centers for Disease Control and Prevention. each step were $0.722,0.648,0.647$, and 0.456 , respectively (Fig. 2). The AUC values of each criterion of the KTAS $(\mathrm{KTAS} \leq 1, \mathrm{KTAS} \leq 2, \mathrm{KTAS} \leq 3, \mathrm{KTAS} \leq 4$, and KTAS $\leq 5)$ were $0.550,0.745,0.597,0.501$, and 0.50 respectively (Fig. 3 ).

\section{DISCUSSION}

This study was designed to evaluate the suitability of the trauma triage systems that are currently used in Korea, including the CDC field triage system and the KTAS.

At the prehospital stage, it is necessary to determine whether the patient is a major trauma patient in order to commence transfer to a trauma center within a short period of time with limited information. Because of the

Table 2. The undertriage/overtriage rates of each KTAS criterion

\begin{tabular}{|lccccc|}
\hline & KTAS $\leq 1$ & KTAS $\leq 2$ & KTAS $\leq 3$ & KTAS $\leq 4$ & KTAS $\leq 5$ \\
\hline Undertriage & $147(15.2)$ & $55(7.4)$ & $9(4.1)$ & $0(0.0)$ & $0(0.0)$ \\
Overtriage & $21(50.0)$ & $153(57.5)$ & $632(79.9)$ & $839(83.3)$ & $841(83.3)$ \\
\hline
\end{tabular}

Values are presented as number (\%).

KTAS: Korean Triage and Acuity Scale.

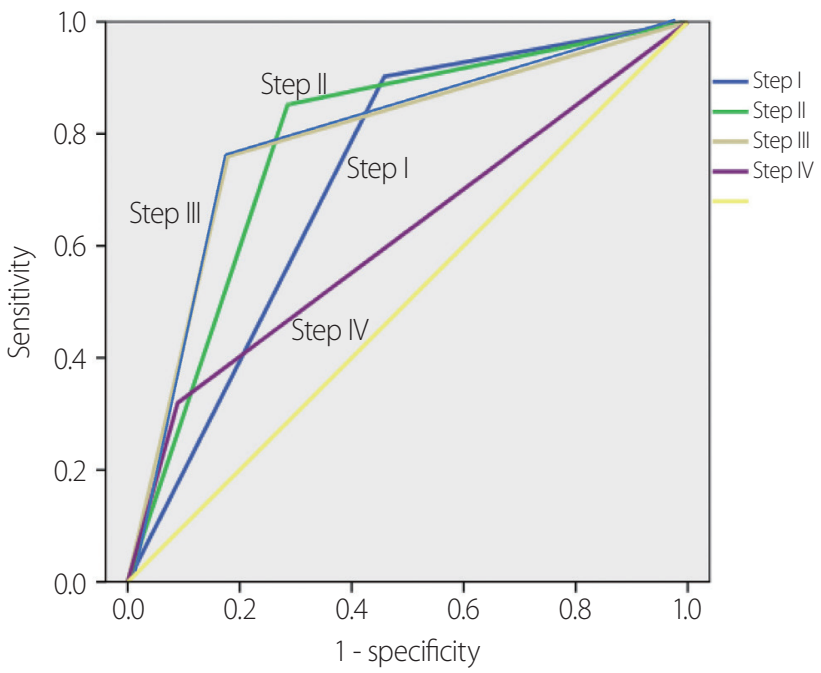

Fig. 1. The ROC curve of each step (steps I, II, III, and IV) of the CDC triage system. The AUC values of each step (steps I, II, III, and IV) of the CDC triage system were $0.722,0.783,0.791$, and 0.615 , respectively. ROC: receiver operating characteristic, CDC: Centers for Disease Control and Prevention, AUC: area under the curve. 
complexity of this task, various algorithms have been developed for trauma field triage. Most trauma triage algorithms have been made using a combination of variables reflecting several components, such as physiologic elements, anatomic/physical injuries, injury mechanisms/kinetic elements, patient factors, and other considerations, such as the decision of EMS personnel and prehospital resuscitation $[1,3,4,6,8]$.

Fig. 2 shows how physiologic factors contributed the most to the performance of the CDC triage system in this study, which is consistent with the results of previous research [11,12]. Physiologic categories are also one of the most important factors in determining the severity of trauma. This suggests that physiologic factors must be included in a modified or newly developed algorithm and should also be considered as the initial step.

Based on our results, we conclude that it is better to omit step IV of the CDC triage system because the overtriage rate markedly increased from $59.4 \%$ in step III to $78.9 \%$ in step IV, and, moreover, the undertriage rate also increased from $4.5 \%$ to $5.3 \%$ (Table 1, Fig. 1). Fig. 2 shows the adverse effect of the component of special considerations on the performance of the entire triage system.

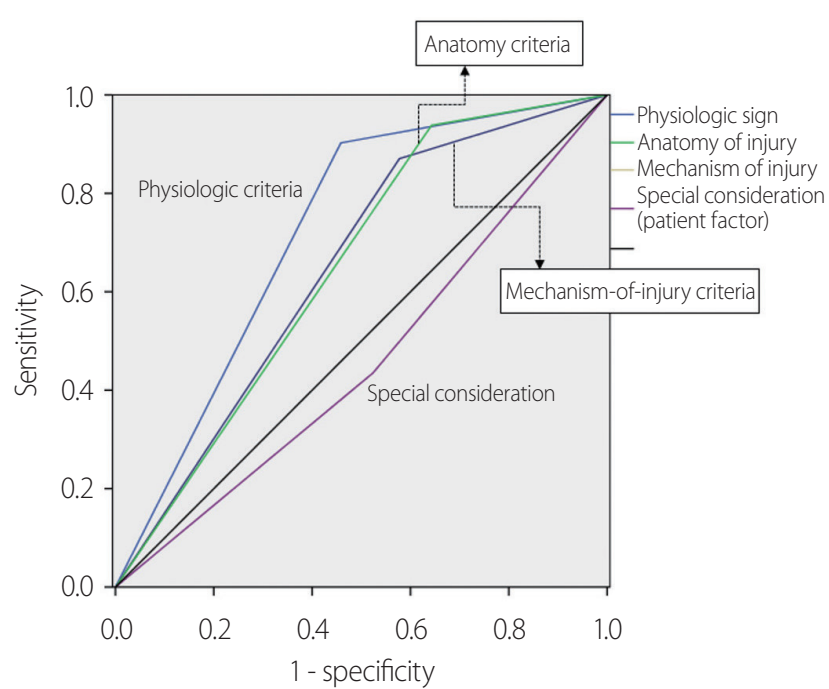

Fig. 2. The ROC curve for separate components of each step (physiologic criteria, anatomic criteria, mechanism-of-injury criteria, and special considerations). The AUC values for the separate components of each step (physiologic criteria, anatomic criteria, mechanism-of-injury criteria, and special considerations) were $0.722,0.648,0.647$, and 0.456 , respectively. ROC: receiver operating characteristic, AUC: area under the curve.
The KTAS showed relatively good results for initial triage. A KTAS score equal or less than 3 reflects a similar outcome to step III of the CDC triage system. The KTAS is currently in wide use as an initial triage system in trauma centers. Trauma patients whose KTAS scores are $\leq 2$ or 3 should be treated in the trauma bay.

Both triage protocols yielded relatively good undertriage rates, but the overtriage rates of both triage protocols were disappointing. The overtriage rate of the CDC triage system was better than that of the KTAS.

If we only consider the outcome in terms of the overtriage/undertriage rate, the results of the CDC triage system were acceptable. It seemed to be a good indicator of major trauma in steps II and III. However, there is a possible problem at the stage of collecting information on patient variables. On one hand, physiologic signs, such as the GCS, and directly observed variables, such as amputation, were well documented. On the other hand, values were recorded in fewer than $1 \%$ of cases for variables that are determined through a physical examination or by a detailed history-taking, such as flail chest, high-risk auto/ vehicular accident, and past medical history. This suggests a flawed assessment of these variables. In particular, the

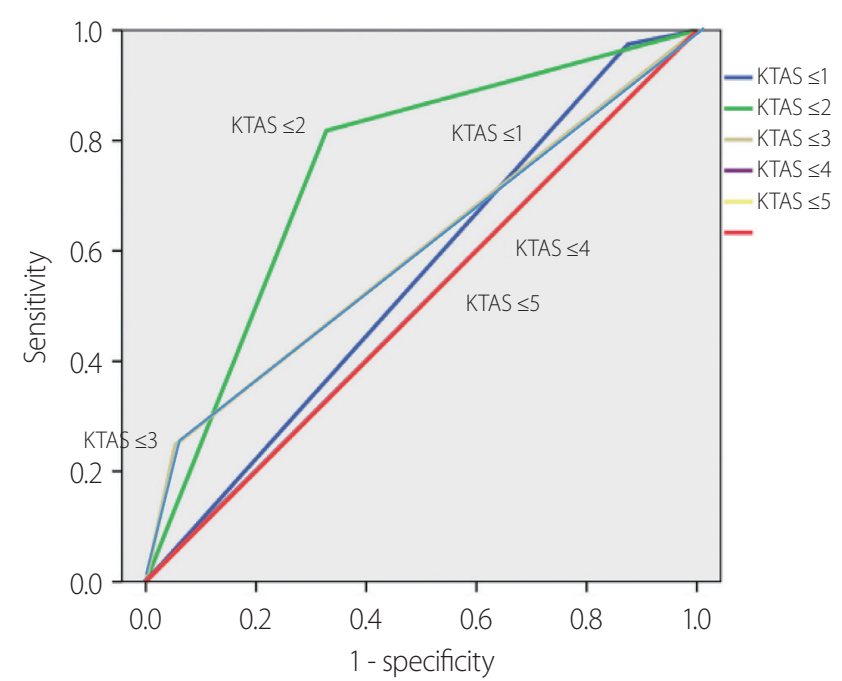

Fig. 3. The ROC curves of each KTAS criterion (KTAS $\leq 1, \mathrm{KTAS} \leq 2$, KTAS $\leq 3, \mathrm{KTAS} \leq 4$, and KTAS $\leq 5$ ). The AUC values of each criterion of KTAS (KTAS $\leq 1, \mathrm{KTAS} \leq 2, \mathrm{KTAS} \leq 3, \mathrm{KTAS} \leq 4$, and KTAS $\leq 5$ ) were $0.550,0.745$, $0.597,0.501$, and 0.50 , respectively. The lines of KTAS $\leq 3$ and KTAS $\leq 4$ were so close that they seemed to overlap with each other. KTAS: Korean Triage and Acuity Scale, ROC: receiver operating characteristic, AUC: area under the curve. 
documentation for step IV was likely to be incorrect based on the rare incidence of the variables evaluated at this step, except for those related to age. This may be a crucial reason for the poor performance of step IV.

On scene, estimating the velocity of a crash and the depth of intrusion is virtually impossible. Vehicle telemetry is not mounted on Korean cars. A variable that is unnecessary or difficult to assess should be eliminated.

This study has three main limitations. First, it was retrospective in nature. Second, every variable should have been collected from the EMS notes, but this was not the case. We were obligated to collect data from our medical records because we either could not access the EMS notes or could obtain enough information from them. However, we think that the data on-scene were not significantly different from the hospital data because the transfer time is short in Incheon, Korea. Finally, this was a single-center study. However, our regional trauma center is located in the third largest city of Korea and has one of the largest volume of patients in the nation. Therefore, we think that our data can be considered representative of Korean trauma care to a meaningful degree.

\section{CONCLUSION}

In conclusion, the CDC field triage system is acceptable, but not ideal, for Korean trauma care. If we follow the protocol, it would be preferable to omit step IV. The KTAS seems to be a good indicator for in-hospital triage. However, ultimately, a new triage protocol that is simple to execute on-scene and simultaneously has good performance should be developed.

\section{REFERENCES}

1. Cameron PA, Gabbe BJ, Smith K, Mitra B. Triaging the right patient to the right place in the shortest time. Br J Anaesth 2014;113:226-33.

2. Mackersie RC. History of trauma field triage development and the American College of Surgeons criteria. Prehosp Emerg Care 2006;10:287-94.

3. Sasser SM, Hunt RC, Faul M, Sugerman D, Pearson WS, Dulski T, et al. Guidelines for field triage of injured patients: recommendations of the National Expert Panel on Field Triage, 2011. MMWR Recomm Rep 2012;61:1-20.

4. Hornez E, Maurin O, Mayet A, Monchal T, Gonzalez F, Kerebel D. French pre-hospital trauma triage criteria: does the "pre-hospital resuscitation" criterion provide additional benefit in triage? World J Crit Care Med 2014;3:68-73.

5. Moore L, Lavoie A, Abdous B, Le Sage N, Liberman M, Bergeron E, et al. Unification of the revised trauma score. J Trauma 2006;61:718-22; discussion 722 .

6. van Laarhoven JJ, Lansink KW, van Heijl M, Lichtveld RA, Leenen LP. Accuracy of the field triage protocol in selecting severely injured patients after high energy trauma. Injury 2014;45:869-73.

7. Korean National Fire Agency. The standard protocols for 119 emergency medical services providers [Internet]. Sejong: Korean National Fire Agency 2019 [cited 2019 Nov 25]. Available from http://www.nfa.go.kr/nfa/publicrelations/legalinformation/0017/0003/?boardId=bbs_0000000000001097\&mode $=$ view $\&$ cntId $=4 \&$ categor $y=\&$ pageId $x=\&$ searchCondition $=\&$ searchKeyword $=$.

8. Park J, Lim T. Korean triage and acuity scale (KTAS). J Korean Soc Emerg Med 2017;28:547-51.

9. Gennarelli TA, Wodzin E. The Abbreviated Injury Scale 2005. Update 2008. Des Plaines:American Association for Automotive Medicine;2008.

10. American College of Surgeons Committee on Trauma. Resources for optimal care of the injured patient. Chicago:Google Scholar;2016.

11. Brown JB, Stassen NA, Bankey PE, Sangosanya AT, Cheng JD, Gestring ML. Mechanism of injury and special consideration criteria still matter: an evaluation of the National Trauma Triage Protocol. J Trauma 2011;70:38-44; discussion 44-5.

12. Cassignol A, Marmin J, Cotte J, Cardinale M, Bordes J, Pauly $\mathrm{V}$, et al. Correlation between field triage criteria and the injury severity score of trauma patients in a French inclusive regional trauma system. Scand J Trauma Resusc Emerg Med 2019;27:71. 